\title{
Application d'un produit polymère à la consolidation des sables carbonatés
}

\section{A. BENNABI}

Laboratoire de Mécanique et de Géomécanique, Université de Nantes,

2, rue de la Houssinière, 44072 Nantes cedex

\section{LEVACHER}

Laboratoire de Mécanique des Fluides et de Génie Civil, Université du Havre, quai Frissard, BP 265, 76055 Le Havre cedex
L'injection est un procédé de construction de plus en plus utilisé pour l'étanchement ou la consolidation des sols. Le choix du produit d'injection dépend des caractéristiques du sol et de l'objectif du traitement. Les coulis de ciment. injectés sous pression, sont couramment utilisés. Cependant, l'utilisation de produits chimiques peut être plus avantageuse grâce, principalement, à leur faible viscosité assurant une meilleure pénétrabilité des terrains à injecter.

Cet article décrit les travaux réalisés avec un nouveau produit polymère utilisé pour la consolidation des sables carbonatés. Ces formations présentent des

caractéristiques mécaniques faibles à très faibles, et le traitement envisagé permet d'augmenter leur résistance mécanique.

L'expérimentation, utilisant en grande partie l'essai de compression simple, porte sur des échantillons de sable préalablement injectés. Des valeurs de la résistance de 3,5 à $4 \mathrm{MPa}$ sont régulièrement obtenues dès quelques heures de conservation dans l'eau. L'amélioration de la résistance mécanique est étudiée en fonction des principaux paramètres liés aux problèmes d'injection.

\section{Application of a polymer product to the consolidation of calcareous sands}

Grouting is an increasingly usual construction procedure which both reduces permeability and increases strength of soils. The choice of the appropriate grout depends on the properties of the material to be injected and on the desired effect. Cement grouts injected under pressure are widely used. But the use of chemical solutions can be more appropriate as their low viscosity enables them to penetrate very small pores.

In the research work presented here, the grout is a new polymer which is used for the consolidation treatment of calcareous sands. These formations have weak mechanical characteristics. The grouting consolidation treatment consists in their strengthening.

The investigation is mainly based on unconfined compression tests performed on samples previously grouted. Increases in strength are observed : values of 3,5 and $4 \mathrm{MPa}$ for the unconfined compression strength are commonly reached for a curing time in water of anywhere a few hours to some days. The strength of the samples is studied in function of the main parameters which concern the grouting. 


\section{Introduction}

Une première étude expérimentale a été réalisée avec un nouveau produit polymère pour tester son efficacité dans l'injection des pieux battus dans les sables carbonatés [1]. Rappelons que le frottement latéral des pieux battus dans ces formations est très faible devant les valeurs obtenues dans les sables siliceux. Dans cette première étude il s'agissait, d'une part, de définir les paramètres d'injection permettant la réalisation de la nécessaire condition d'adhérence entre le pieu métallique et le bulbe de sable injecté et, d'autre part, de vérifier le gain de frottement latéral des pieux battus en cuve d'essai, obtenu après injection.

L'efficacité de l'injection de ce nouveau polymère étant démontrée en laboratoire, nous avons procédé à la réalisation d'une nouvelle étude expérimentale [2] portant sur la caractérisation mécanique dé l'injection du sable carbonaté à l'aide de ce polymère. Cette caractérisation est obtenue grâce à l'essai de compression simple mené sur des éprouvettes de sable injecté.

\section{2.}

\section{Caractérisation de l'injection}

\section{1}

\section{Méthodologie expérimentale}

\section{1,1}

Produit

Le produit utilisé, fourni par la société GTI [3] est un copolymère acrylique comportant des fonctions acides. Il est soluble dans l'eau. Sa viscosité dynamique et sa densité sont faibles. Différentes teneurs en extrait sec ont été considérées. La figure 1 montre l'évolution de la viscosité en fonction de la température pour une teneur en extrait sec (ES) de $30 \%$. On rappelle que la viscosité de l'eau pure est de $1 \mathrm{mPa}$.s.

Les valeurs de la densité sont de 1,051; 1,075 et 1,130 pour des teneurs en extrait sec de $15 \%, 25 \%$ et $30 \%$ respectivement.
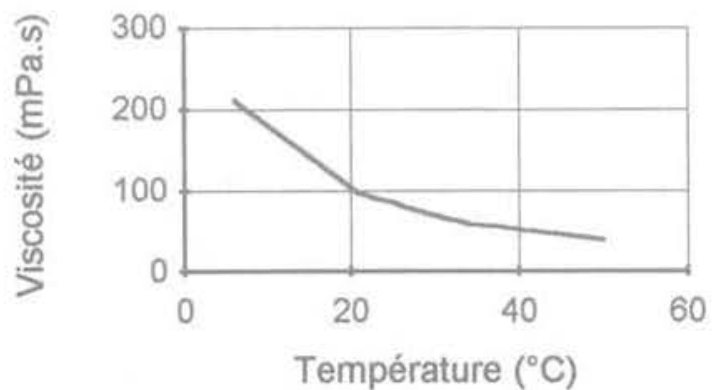

FG.1 Viscosité du produit polymère (ES $=30 \%$ ).

Polymer viscosity (Dry Matter Content $=30 \%$ ).

\subsection{2}

\section{Matériaux}

Deux principaux matériaux ont été utilisés :

- le sable naturel de Manche, de $90 \%$ de teneur en $\mathrm{CaCO}_{3}$. Différentes classes granulométriques ont été utilisées :

- un sable artificiel obtenu par concassage d'une roche métamorphique d'origine pyrénéenne, de teneur en $\mathrm{CaCO}_{3}$ avoisinant les $100 \%$. Ce matériau était livré dans diverses classes granulométriques par la société OMYA [4].

\subsection{3}

\section{Préparation des éprouvettes de sable injecté}

\section{- Première phase : éprouvette de sable (Fig. 2)}

Le sable est versé dans un tube cylindrique en PVC transparent de $56 \mathrm{~mm}$ de diamètre. Sa mise en place s'effectue par dépôt à hauteur de chute constante. La figure 2 illustre le procédé utilisé : le sable est déversé depuis un entonnoir d'un goulot de $4 \mathrm{~mm}$ de diamètre, et la hauteur de chute, de $20 \mathrm{~cm}$, est contrôlée à l'aide d'une tige fixée sur l'entonnoir, grâce à la transparence du moule en PVC. L'extrémité inférieure de la tige se trouve, à tout moment, au niveau de la surface de dépôt du sable.

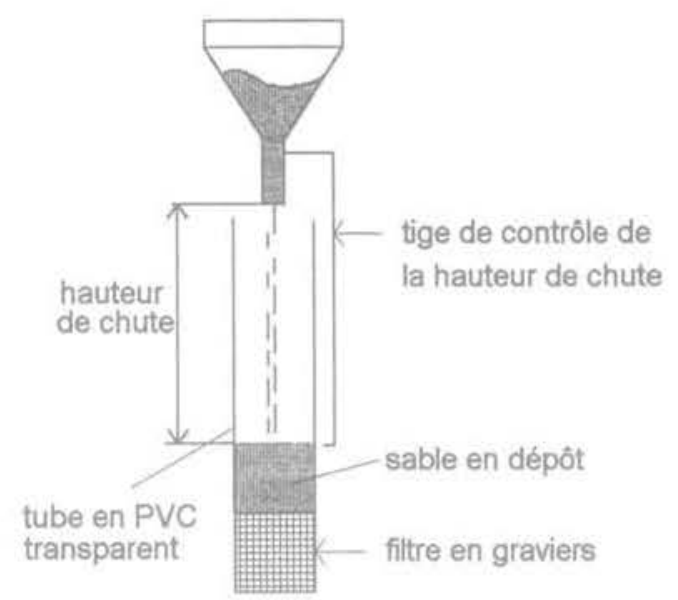

FGa.2 Préparation de l'éprouvette de sable. Sand sample preparation.

L'opération de dépôt est accompagnée d'un déplacement manuel continu sur toute la section de l'échantillon, de sorte que la surface de dépôt reste plane pendant la mise en place du sable.

Le sable est ainsi mis en place entre deux filtres de graviers (de type 5/8) qui permettront au produit de s'écouler à travers l'échantillon sans problème de discontinuité aux extrémités de ce dernier.

\section{- Deuxième phase : injection (Fig. 3)}

Une fois l'éprouvette de sable constituée, la phase de l'injection intervient. Un certain nombre d'éprouvettes ont été injectées à l'état sec, mais la plupart ont d'abord été humidifiées. L'humidification, par envoi d'eau sans application de pression, est réalisée du bas vers le haut de l'échantillon. Cette opération est arrêtée lorsque l'eau arrive au sommet du filtre supérieur. 


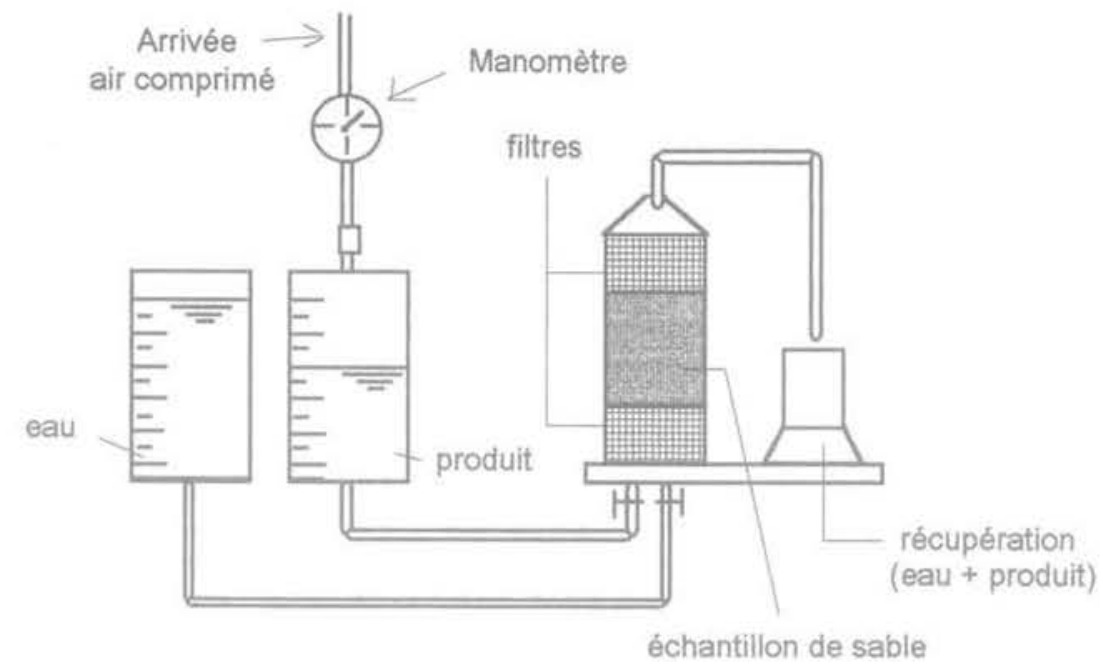

FG, 3 Procédure d'injection.

Injection procedure.

Le produit polymère est alors injecté sous pression. Il pénètre dans l'échantillon de sable, par le bas, en repoussant l'eau qui se trouve dans les interstices. L'injection est terminée lorsque le produit apparait au niveau du filtre supérieur.

Les échantillons de sable ainsi injectés sont ensuite maintenus dans leurs moules jusqu'à l'obtention d'une certaine résistance permettant de les retirer sans risquer de les abîmer. Après divers essais, le temps de maintien dans le moule a généralement été fixé à 1 heure.

Au démoulage, on procède à un certain nombre de mesures permettant de déterminer les quantités d'eau et de produit mises en jeu, ainsi que les principales caractéristiques physiques des échantillons de sable (poids volumique moyen, volume des vides, teneur en eau...).

Les éprouvettes de sable injecté sont conservées dans des conditions (dans l'air ou dans l'eau) et pendant une durée (de quelques minutes à quelques jours) déterminées.

Une première partie des essais de compression simple $\left(n^{\circ} 1\right.$ à $\left.n^{\circ} 17\right)$ a été réalisée avec des éprouvettes de $56 \mathrm{~mm}$ de diamètre. Avant l'essai elles étaient taillées par sciage, et leur hauteur était ramenée à $112 \mathrm{~mm}$ (élancement 2). La réalisation de ces essais se heurtait quelquefois à la difficulté d'obtenir des sections droites parfaitement planes et parallèles. Une modification de la procédure de préparation des éprouvettes a alors été apportée et a consisté à éliminer l'opération de sciage. Les éprouvettes étaient aux dimensions requises pour l'essai de compression simple dès le démoulage. A partir de cette modification, le diamètre était de $45 \mathrm{~mm}$ et la hauteur de $90 \mathrm{~mm}$.

\section{2}

\section{Description sommaire de la réaction chimique}

Le produit d'injection est un polymère soluble dans l'eau qui comporte des radicaux actifs judicieusement placés le long de la chaîne macromoléculaire.

Lorsque ce produit est injecté dans un sol, dès qu'il rencontre un site carbonaté, les radicaux actif́s réagis- sent sur les sites $\mathrm{CO}_{3}$ et se fixent donc sur le minéral en dégageant du $\mathrm{CO}_{2}$ et de l'eau. La chaîne macromoléculaire étant suffisamment longue, elle réagit par différents sites répartis le long de la macromolécule sur plusieurs grains de carbonates et assure donc une liaison chimique entre ces derniers.

Le résultat de cette injection est une cimentation artificielle des formations carbonatées.

Le développement des caractéristiques mécaniques au sein de ces « nouveaux matériaux » est fonction de nombreux paramètres qui régissent les phénomènes se produisant pendant la réaction chimique et pendant le durcissement : nature des matériaux (faiblement ou fortement carbonatés), dimension des grains présentant une plus ou moins grande surface spécifique, densité et porosité des matériaux, viscosité et teneur en extrait sec du produit, conditions de conservation des éprouvettes injectées.

La figure 4 montre schématiquement les différentes phases de la préparation des éprouvettes.

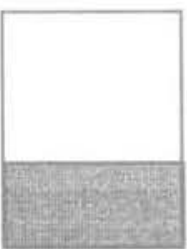

(1)

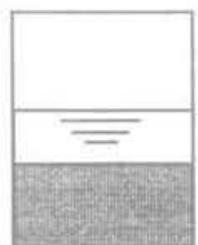

(2)

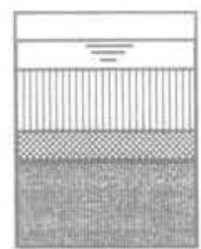

(3)
Grains solides

$\square$ Air
$\square$ Eau
Polymère amorphe
Complexe grains/polymère

(1) : Fabrication de l'échantilon sec

(2) : Humidification

(3) : Injection

16.4 Différentes phases de la préparation des éprouvettes.

Different steps of samples preparation. 
Dans la phase (3), en plus des grains solides, de l'eau et de l'air, il y a deux nouveaux constituants : le complexe grains/polymère qui se trouve au niveau des contacts intergranulaires et le polymère amorphe qui n'a pas réagi du fait de son éloignement des sites actifs.

\section{3}

\section{Étude de la résistance à la compression simple}

Cette étude a été réalisée sous la forme d'une exploration des propriétés mécaniques du sable injecté, ce qui explique le nombre relativement élevé des paramètres retenus :

- la pression d'injection,

- la densité du sable,

- la durée de conservation des éprouvettes,

- la granulométrie,

- la vitesse de l'essai de compression simple,

- la forme des particules.

\subsection{1}

\section{Résistance en fonction de la pression d'injection}

Le tableau I présente les conditions de réalisation des essais et les résultats de l'essai de compression simple. Le matériau utilisé était le sable de Manche tamisé à $2 \mathrm{~mm}$. Les éprouvettes étaient démoulées 45 minutes après la fin de l'injection et stockées pendant 24 heures dans l'eau à $20^{\circ} \mathrm{C}$.

\section{TABLEAUI}

\begin{tabular}{l|c|c|c}
\multicolumn{1}{c|}{ Numéro de l'essai } & 1 & 2 & 3 \\
\hline $\begin{array}{l}\text { Poids volumique moyen } \\
\text { avant injection (kN/m') }\end{array}$ & 12,6 & 12,4 & 12,3 \\
\hline Indice de densité & 0,33 & 0,29 & 0,21 \\
\hline Indice des vides e & 1,14 & 1,18 & 1,19 \\
\hline Porosité (\%) & 53,3 & 54,1 & 54,4 \\
\hline Extrait sec du produit ES (\%) & 15 & 15 & 15 \\
\hline Pression d'injection (kPa) & 70 & 90 & 110 \\
\hline \begin{tabular}{l|c|c} 
Volume injecté (cm $\left.{ }^{3}\right)$ \\
\hline Durée de stockage (heures)
\end{tabular} & 24 & 24 & 24 \\
\hline $\begin{array}{l}\text { Vitesse de déformation } \\
\text { (mm/mn) }\end{array}$ & 0,65 & 0,65 & 0,65 \\
\hline $\begin{array}{l}\text { Résistance à la compres- } \\
\text { sion simple (MPa) }\end{array}$ & 2,03 & 1,84 & 1,58 \\
\hline $\begin{array}{l}\text { Déformation } \\
\text { à la rupture (\%) }\end{array}$ & 1,53 & 1,60 & 2,44 \\
\hline
\end{tabular}

Une injection à faible pression permet au produit d'enrober correctement les grains de carbonates. Les radicaux actifs du produit réagissent avec un nombre élevé de sites $\mathrm{CO}_{3}$ et forment ainsi autant de liaisons intergranulaires. Lorsque la pression d'injection diminue, la résistance à la compression simple Rc augmente

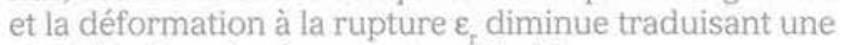
rigidification des éprouvettes (Fig. 5).

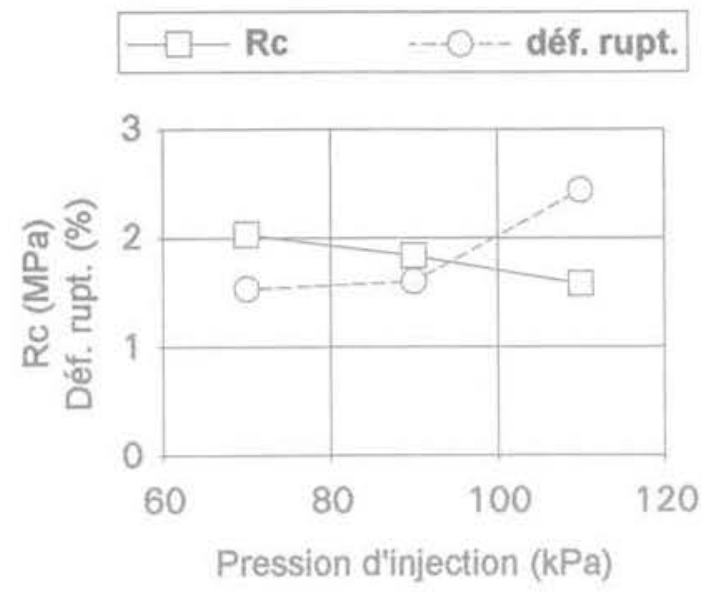

FG 5 Résistance à la compression simple et déformation à la rupture en fonction de la pression d'injection.

Effect of grouting pressure on strength and failure strain.

\section{2:3.,}

Résistance en fonction de l'état de compacité du sable

Le tableau II présente les conditions d'essais et les résultats obtenus pour trois échantillons injectés sous une pression de $90 \mathrm{kPa}$ avec un produit à $15 \%$ d'extrait sec.

\section{TRBLEAU it}

\begin{tabular}{l|c|c|c}
\multicolumn{1}{c|}{ Numéru de lessai } & 4 & 5 & 6 \\
\hline $\begin{array}{l}\text { Poids volumique moyen } \\
\text { avant injection (kN/m })^{3}\end{array}$ & 12,4 & 13,5 & 13,8 \\
\hline Indice de densité & 0,29 & 0,63 & 0,73 \\
\hline Indice des vides e & 1,18 & 1,00 & 0,96 \\
\hline Porosité (\%) & 54,0 & 50,0 & 48,9 \\
\hline Extrait sec du produit ES (\%) & 15 & 15 & 15 \\
\hline Pression d'injection (kPa) & 90 & 90 & 90 \\
\hline \begin{tabular}{l|c|c} 
Volume injecté (cm $\left.{ }^{3}\right)$ \\
\hline Durée de stockage (heures)
\end{tabular} & 24 & 24 & 24 \\
\hline $\begin{array}{l}\text { Vitesse de déformation } \\
\text { (mm/mn) }\end{array}$ & 0,65 & 0,65 & 0,65 \\
\hline $\begin{array}{l}\text { Résistance à la compres- } \\
\text { sion simple (MPa) }\end{array}$ & 1,85 & 2,44 & 2,96 \\
\hline $\begin{array}{l}\text { Déformation } \\
\text { à la rupture (\%) }\end{array}$ & 1,60 & 1,50 & 1,80 \\
\hline
\end{tabular}

La figure 6 regroupe les résultats concernant la résistance à la compression simple et le module de déformation pour ces échantillons. Les valeurs de ces deux paramètres augmentent avec la densité du sable, ce qui peut s'expliquer par le fait que de deux échantillons de sable de mêmes volumes, celui qui présente une plus grande densité comptabilise un nombre de grains plus important et, s'ils sont injectés dans les mêmes conditions, développera un plus grand nombre de liaisons, d'où la rigidité plus grande de l'éprouvette. 


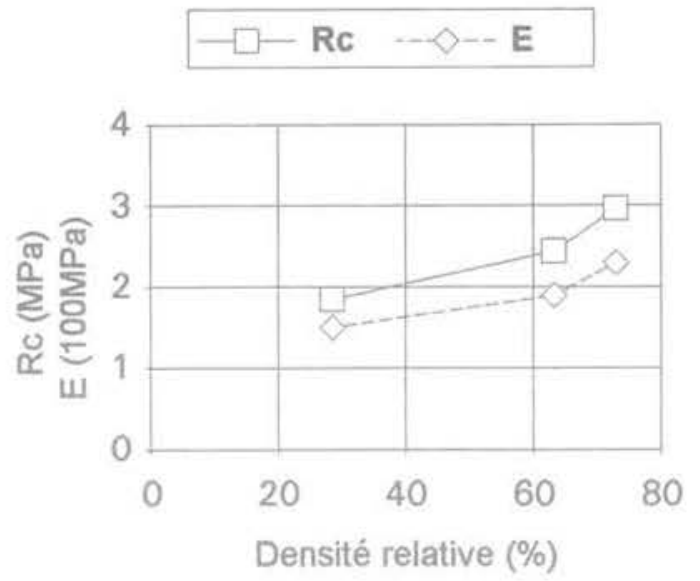

FG.6 Résistance à la compression simple et module de déformation en fonction de l'état de compacité du sable.

Effect of density on strength and on strain modulus.

La figure 7 montre l'allure générale des courbes contrainte-déformation obtenues pour ces mêmes échantillons.

Pour les éprouvettes constituées de sable lâche, la forme étalée du maximum est révélatrice d'une rupture de type plastique. Le sommet nettement moins arrondi des courbes relatives aux éprouvettes denses traduit un comportement de type radoucissant à la rupture.

\section{2:35}

\section{Résistance en fonction du temps}

Deux séries d'essais composent cette étude. La première série d'essais permet d'obtenir des informations sur le temps de prise. En effet, il n'existe pas de
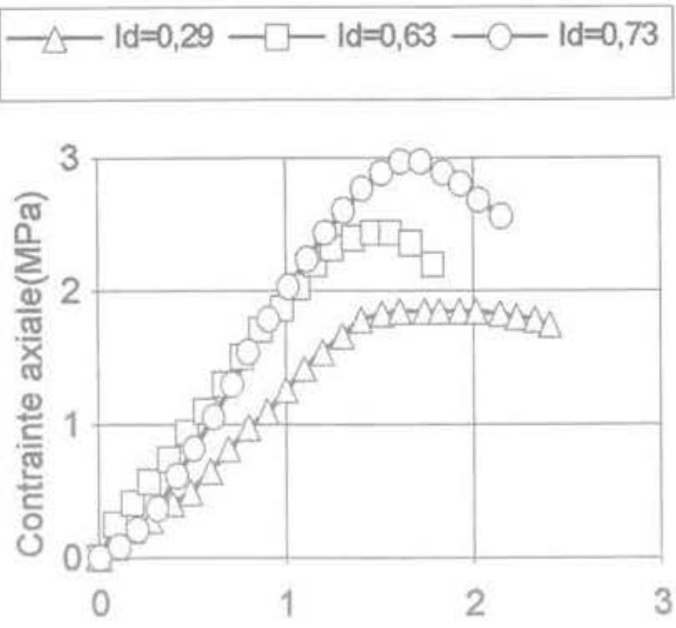

Déformation axiale (\%)

FQ.7 Courbes contrainte-déformation paramètre " densité du sable $"$. Typical « stress-strain » curves (sand density parameter).

méthode standard pour la détermination du temps de prise des produits chimiques. Pour certains produits, plusieurs auteurs mesurent, dans une éprouvette inclinée, le temps nécessaire à l'arrêt de l'écoulement. Le produit utilisé dans ces travaux ne faisant prise qu'en présence de carbonates, ces méthodes sont inopérantes. L'essai de compression simple a alors été utilisé pour obtenir une estimation du temps de prise. En réalité, ce qui est mesuré, c'est le temps nécessaire à l'obtention d'une résistance non négligeable. La deuxième série d'essais étudie l'évolution de la résistance des éprouvettes en fonction du temps de stockage dans l'eau. Le tableau III précise les conditions générales de cette étude, effectuée avec un produit à

\section{TABLEAU III}

\begin{tabular}{|c|c|c|c|c|c|c|c|c|c|c|c|}
\hline Numéro de l'essaí & 7 & 8 & 9 & 10 & 11 & 12 & 13 & 14. & 15 & 16 & 17 \\
\hline $\begin{array}{l}\text { Poids volumique moyen } \\
\text { avant injection }\left(\mathrm{kN} / \mathrm{m}^{3}\right)\end{array}$ & 12,9 & 12,5 & 13,0 & 12,9 & 12,7 & 12,3 & 12,7 & 12.7 & 12,9 & 12,9 & 12,7 \\
\hline Indice des vides e & 1,09 & 1,17 & 1,08 & 1,10 & 1,13 & 1,20 & 1,13 & 1,13 & 1,10 & 1,10 & 1,13 \\
\hline Extrait sec du produit ES (\%) & \multicolumn{11}{|c|}{30} \\
\hline Pression d'injection $(\mathrm{kPa})^{*}$ & 150 & 150 & 180 & 165 & 170 & 180 & 150 & 150 & 160 & 160 & 160 \\
\hline Volume injecté $\left(\mathrm{cm}^{3}\right)^{* *}$ & \multicolumn{11}{|c|}{$39 \pm 3$} \\
\hline $\begin{array}{l}\text { Temps de maintien dans } \\
\text { le moule (mn) }\end{array}$ & 60 & 55 & 30 & 33 & 25 & 22 & \multicolumn{5}{|c|}{35} \\
\hline Durée de stockage (heures) & 1 & 1 & 1 & 1 & 1 & 1 & 2,75 & 15 & 264 & 0,77 & 2,13 \\
\hline $\begin{array}{l}\text { Vitesse de déformation } \\
(\mathrm{mm} / \mathrm{mn})\end{array}$ & \multicolumn{11}{|c|}{0,50} \\
\hline $\begin{array}{l}\text { Résistance à la compres- } \\
\text { sion simple (MPa) }\end{array}$ & 1,96 & 1,30 & 0,79 & 0,84 & 0,71 & 0,51 & 2,00 & 4,48 & 3,57 & 2,59 & 2,98 \\
\hline $\begin{array}{l}\text { Déformation } \\
\text { à la rupture }(\%)\end{array}$ & 1 & I & 4,5 & 4,4 & 4,3 & 4,5 & 1 & 1,50 & 1,50 & 2,50 & 1,95 \\
\hline
\end{tabular}

* Dans cette série d'essais la pression d'injection était difficile à maitriser.

** Il s'agit du volume restant dans les interstices à la fin de l'injection (le volume envoyé sous pression est de $100 \pm 3 \mathrm{~cm}^{3}$ ). 
$30 \%$ d'extrait sec sur, d'une part, des éprouvettes de sable de Manche tamisé à $2 \mathrm{~mm}$ et écrasées tout de suite après le démoulage et, d'autre part, sur des éprouvettes de sable de Manche tamisé à 1,6 $\mathrm{mm}$ et conservées dans l'eau pendant des durées variant de quelques heures à quelques jours.

La figure 8 montre les valeurs obtenues de la résistance à la compression simple. La résistance augmente durant les premières heures puis semble se stabiliser assez rapidement autour d'une valeur de $3,5 \mathrm{MPa}$. Le contact du polymère avec le carbonate de calcium entraîne une réaction chimique qui crée des liaisons entre les grains de sable.

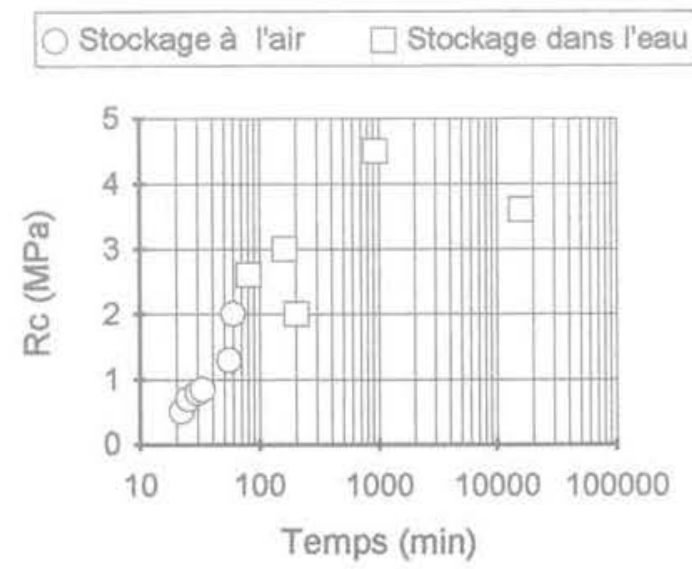

भis. : Résistance à la compression simple en fonction du temps.

Effect of time on unconfined compressive strength $(O:$ storage in air, $\square:$ storage into water).

Tant que cette réaction se poursuit: le nombre de liaisons et la résistance qui en résulte, augmentent. Il est possible de lier les quelques écarts relativement importants de la résistance à la compression simple à la dispersion produite par la méthode de préparation des échantillons. En effet, la fabrication des échantillons de sable, par versement de ce dernier dans un moule, est une opération manuelle qui, même conduite avec le

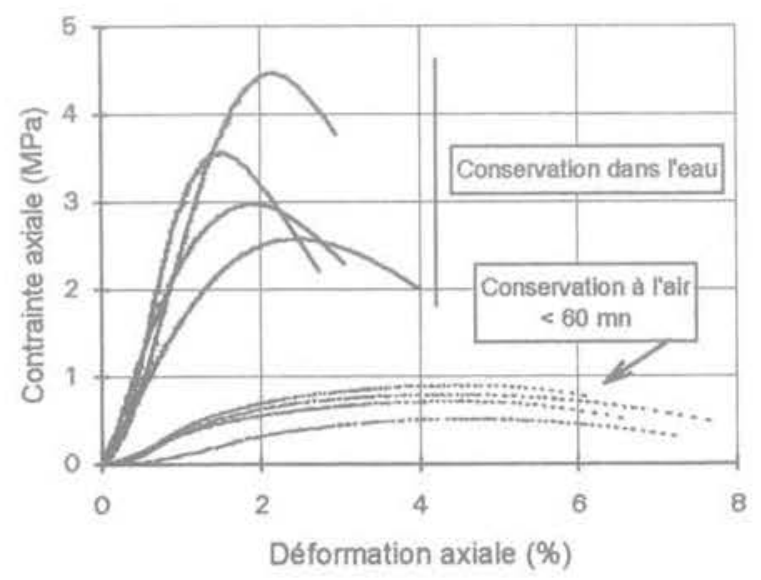

FIG.9 Courbes contrainte-déformation à différentes durées de conservation. Stress-strain curves at different curing time. plus grand soin, induit nécessairement des différences de structure au sein de l'échantillon.

Les courbes contrainte-déformation obtenues pour la plupart de ces éprouvettes sont représentées sur la figure 9.

La comparaison des courbes de la figure 9 montre l'évolution du comportement des éprouvettes, testées à l'essai de compression simple, quelques dizaines de minutes à plusieurs jours après leur injection. Les courbes des jeunes échantillons sont caractérisées par un maximum étalé, révélateur d'un comportement plus ou moins plastique. La valeur moyenne obtenue pour la déformation à la rupture tourne autour de 4,5\%. Les valeurs du module initial sont de l'ordre de quelques dizaines de $\mathrm{MPa}$ ( 27 à $57 \mathrm{MPa}$ ). Les courbes obtenues pour des cures plus longues ont des maxima nettement moins étalés. Elles rendent compte d'un comportement à la rupture relativement fragile. Les valeurs de la déformation à la rupture, nettement plus faibles que celles correspondant à la première série, ainsi que les valeurs du module initial, allant de 156 à $400 \mathrm{MPa}$, confirment ce comportement.

La déformation à la rupture diminue avec la durée de conservation. Elle passe d'environ $4,5 \%$ pour les très jeunes échantillons à une valeur de $1,5 \%$ à 15 heures. Des essais de compression simple après des durées de conservation plus importantes ( 1 et 3 mois) n'ont pas donné de valeurs (résistance à la compression simple et déformation à la rupture) significativement différentes de celles relatives aux essais réalisés après quelques jours seulement de stockage

Un nombre réduit d'éprouvettes fait l'objet d'un stockage prolongé. Deux résultats d'essais de compression simple donnent une idée de la stabilisation de la résistance obtenue. Ces résultats ont été obtenus avec des éprouvettes de sable de Manche tamisé à $0,63 \mathrm{~mm}$, injectées à l'aide d'un produit de $25 \%$ de teneur en extrait sec et stockées pendant une durée de 16 mois : les valeurs de la résistance sont de 4,4 MPa et $5 \mathrm{MPa}$ respectivement pour l'éprouvette stockée dans l'eau et pour celle stockée à l'air, le module de déformation est de l'ordre de $600 \mathrm{MPa}$ dans les deux cas.

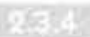

\section{Résistance en fonction de la granulométrie}

Pour la suite de l'expérimentation, les éprouvettes de sable injecté ont un diamètre de $45 \mathrm{~mm}$ et une hauteur de $90 \mathrm{~mm}$.

Les matériaux utilisés dans l'étude de ce paramètre sont les carbonates naturels obtenus par concassage d'une roche métamorphique d'origine pyrénéenne. Les essais ont été réalisés pour des matériaux humides et pour des matériaux secs. Les granulométries considérées sont indiquées dans le tableau IV (en mm) :

\section{TABLEAUIN}

\begin{tabular}{c|c} 
Matériaux humides & Matériaux secs \\
\hline $0,35 / 0,5$ & $0,35 / 0,7$ \\
\hline $0,35 / 0,7$ & $0,6 / 1,6$ \\
\hline $0,6 / 1,6$ & $1 / 2,5$ \\
\hline $1 / 2,5$ & \\
\hline $2 / 4$ & \\
\hline
\end{tabular}




\begin{tabular}{|c|c|c|c|c|c|c|c|c|}
\hline Numéro de lessai & 18 & 19 & 20 & 21 & 22 & 23 & 24 & 25 \\
\hline Granulométrie (mm) & $0,35 / 0,5$ & $0,35 / 0,7$ & $0,6 / 1,6$ & $1 / 2,5$ & $2 / 4$ & $0,35 / 0,7$ & $0,6 / 1,6$ & $1 / 2,5$ \\
\hline Poids volumique moyen avant injection $\left(\mathrm{kN} / \mathrm{m}^{3}\right)$ & 15,2 & 15,6 & 15,3 & 14,9 & 15,3 & 15,9 & 15,6 & 15,2 \\
\hline Indice des vides e & 0,78 & 0,73 & 0,76 & 0,81 & 0,76 & 0,69 & 0,73 & 0,78 \\
\hline Extrait sec du produit ES (\%) & \multicolumn{5}{|c|}{30} & \multicolumn{3}{|c|}{15} \\
\hline Pression d'injection (kPa) & 250 & 170 & 50 & 45 & 70 & 110 & 80 & 40 \\
\hline Volume injecté $\left(\mathrm{cm}^{3}\right)$ & 50 & 57 & 55 & 42 & 20 & 35 & 33 & 25 \\
\hline Temps de maintien dans le moule (mn) & \multicolumn{8}{|c|}{60} \\
\hline Durée de stockage (heures) & \multicolumn{5}{|c|}{48} & \multicolumn{3}{|c|}{96} \\
\hline Vitesse de déformation ( $\mathrm{mm} / \mathrm{mn}$ ) & \multicolumn{8}{|c|}{0,50} \\
\hline $\begin{array}{l}\text { Résistance à la compression simple } \\
\text { (MPa) }\end{array}$ & 4,16 & 3,84 & 1,37 & 0,71 & 0,25 & 1,80 & 1,34 & 0,68 \\
\hline Déformation à la rupture (\%) & 1,70 & 1,55 & 1,30 & 1,05 & 0,70 & 0,50 & 0,90 & 1,10 \\
\hline
\end{tabular}

Le produit utilisé pour les matériaux humides a un extrait sec de $30 \%$. Celui utilisé pour les matériaux secs a été dilué dans l'eau (extrait sec $15 \%$ ). Les échantillons secs présentent après leur injection, comme nous le montrerons plus loin, des résistances plus importantes que les échantillons humides. La dilution du produit permet de rester dans des gammes de résistance courantes.

La quantité de produit envoyée sous pression est constante. Cependant, la quantité effectivement retenue à l'intérieur des échantillons varie en fonction de la plus ou moins grande résistance opposée à l'écoulement du produit. Pour tous les essais, la pression d'injection est la pression minimale permettant l'écoulement du produit, et dans ces conditions la quantité retenue dans l'échantillon augmente avec la finesse des particules constituant ce dernier. Il n'a pas été possible d'injecter une même quantité effective de produit pour tous les échantillons. Le tableau $\mathrm{V}$ indique les conditions dans lesquelles ces essais ont été conduits, ainsi que les résultats de l'essai de compression simple.

Les échantillons injectés à l'état humide ont été stockés pendant 48 heures dans de l'eau à la température de $20^{\circ} \mathrm{C}$. Pour l'échantillon $n^{\circ} 22$, la pression indiquée $(70 \mathrm{kPa})$ a été appliquée à la fin de l'injection. Une pression de $10 \mathrm{kPa}$ a suffi pour injecter près de $80 \%$ de la quantité totale. Mais cette pression un peu trop faible a failli provoquer un « bourrage » de l'échantillon. En effet, le choix de la pression d'injection doit tenir compte de deux inconvénients :

- une pression trop faible permet d'amorcer la réaction chimique, mais est insuffisante pour que l'écoulement du produit puisse se poursuivre. Il se forme alors un bouchon qui s'oppose à la poursuite de l'injection ; - une pression trop importante provoque des phénomènes de boulance et modifie considérablement la structure de l'échantillon.

Ce dernier n'est alors plus homogène, et les résultats ne peuvent être pris en compte dans notre étude.

Les échantillons injectés à l'état sec ont été conservés à l'air, durant 4 jours, à température ambiante $\left(20 \pm 2^{\circ} \mathrm{C}\right)$.
Les courbes « contrainte-déformation » obtenues à l'essai de compression simple sont représentées sur les figures 10 et 11 .

La forme de ces courbes traduit un comportement de type fragile pour les échantillons fins et un comportement quelque peu plastique pour les échantillons de granulométrie plus grossière. L'espace intergranulaire dans les échantillons fins est faible. Le nombre total de contacts intergranulaires dû, d'une part à la finesse des grains et, d'autre part, au nombre plus important des liaisons chimiques, en est responsable.

Lorsque les grains sont plus gros, l'espace intergranulaire est rempli de produit ne réagissant pas chimiquement et donnant lieu à la formation, au sein de l'échantillon, d'une structure plastique.

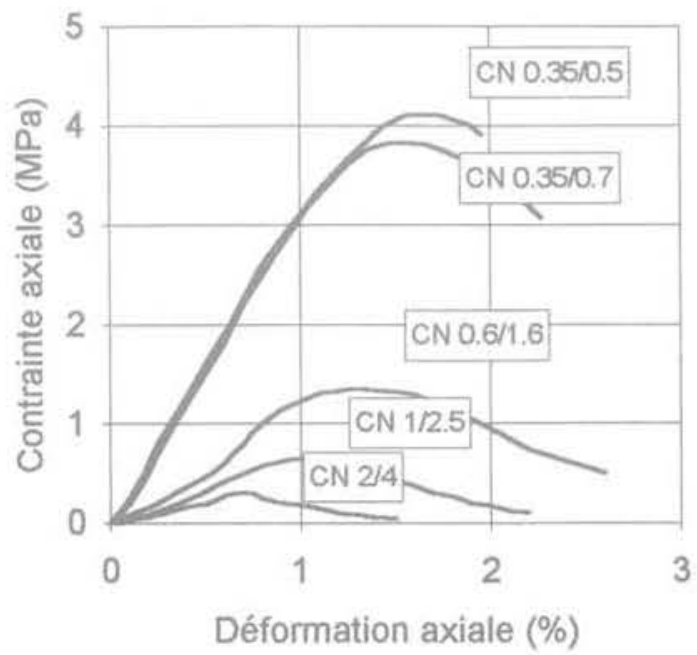

FG. 10 Courbes contrainte-déformation (échantillons de carbonates naturels (CN) humides - produit : $\mathrm{ES}=30 \%$ - stockage : 48 heures dans l'eau).

Stress-strain curves (specimens of wet natural carbonates (CN), polymer : Dry Matter Content $\mathrm{ES}=30 \%$, storage : 48 hours in water). 


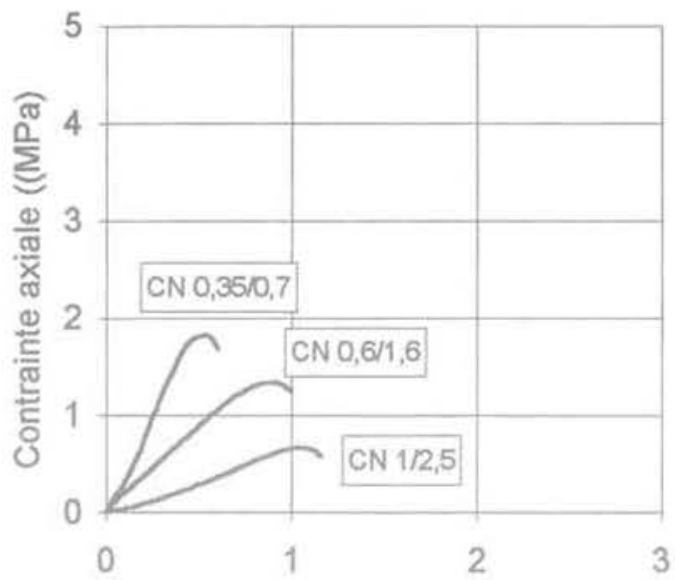

Déformation axiale (\%)

FlG:11 Courbes contrainte-déformation (échantillons secs - produit : $E S=15 \%$ stockage : 4 jours à l'air).

Stress-strain curves (specimens of dry natural carbonates (CN), polymer : Dry Matter Content $\mathrm{ES}=15 \%$, storage : 4 days in air).

La comparaison des courbes obtenues dans le cas des matériaux secs et dans celui des matériaux humides montre que, dans ce dernier cas, la déformation après le pic des contraintes est plus importante. L'interprétation de ce résultat peut être liée au comportement du produit d'injection : en présence d'eau, il se solidifie moins vite et se comporte, pendant l'essai de compression simple, comme une pâte très plastique retenant les grains qui se séparent alors lentement. Dans le cas d'une conservation à l'air, le produit devient plus rigide et les échantillons présentent, en conséquence, un comportement plus fragile.

Concernant la résistance à la compression simple et le module tangent, des tendances similaires sont observées pour les échantillons secs et pour les échantillons humides. En effet, les valeurs de ces paramètres augmentent avec la finesse des grains. Cela est dû au nombre plus imporant des liaisons intergranulaires créées parl'injection du produit polymère. Il en résulte une cohésion beaucoup plus grande dans les échantillons de granulométrie plus fine.

Cependant, les tendances observées pour la déformation à la rupture sont contradictoires. Alors que pour les échantillons secs, la déformation à la rupture diminue avec la finesse des grains, traduisant ainsi un comportement de plus en plus fragile, elle augmente, au contraire, pour les échantillons humides. La présence d'eau permet une plus grande souplesse des liaisons intergranulaires qui, plus nombreuses pour les échantillons fins, peuvent retenir plus longtemps les grains. La valeur, plus élevée, de l'extrait sec, donne en mêrne temps au produit plus de résistance et les liaisons se rompent moins facilement.

La figure 12 montre la forme de la rupture de ces échantillons. Les formes a et b sont rencontrées surtout pour les échantillons résistants. La forme c est celle des échantillons peu résistants.
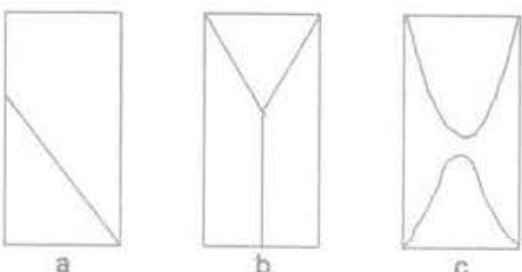

FIG. 12 Rupture des échantillons de carbonates naturels de différentes granulométries. Typical shape of failure of natural carbonates specimens with different grain size distributions.

\section{Mas.t.}

Ébauche d'une méthode de prévision, au moment de l'injection, de la résistance ultérieure des échantillons

Le produit polymère réagit chimiquement avec le carbonate de calcium pour établir des « ponts » entre les grains de carbonates. L'injection du produit polymère dans le sable crée donc des liaisons intergranulaires.

La réaction chimique « polymère-carbonate de calcium ») produit en même temps un dégagement de dioxyde de carbone, $\mathrm{CO}_{\text {, }}$. La quantité dégagée rendant compte de l'intensité de la réaction, il est possible d'imaginer un moyen permettant d'estimer, au moment même de l'injection, la résistance future des échantillons injectés.

La résistance d'un échantillon injecté est liée à sa structure finale : particules solides enrobées et « encastrées $\gg$ dans une matrice de coulis devenue rigide à leur contact. A priori, la résistance obtenue est fonction de la qualité de I'encastrement des particules dans la matrice de coulis qui est elle-même fonction de la dimension et de la forme des particules, de la résistance du coulis dans son état final, ainsi que du nombre de

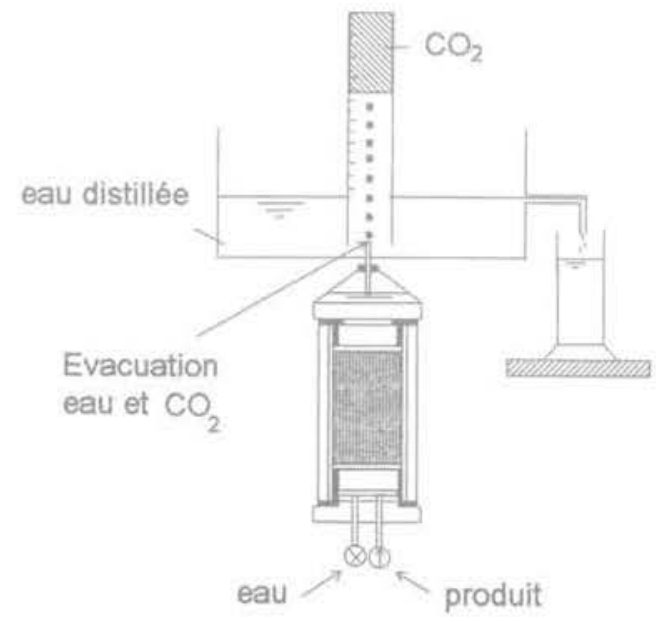

FIG 13 Dispositif de mesure du volume de $\mathrm{CO}$ dégagé pendant la réaction chimique. Device used for the measurement of the release of $\mathrm{CO}_{2}$ during the chemical process. 
liaisons intergranulaires créées entre les particules. Le dégagement de $\mathrm{CO}_{2}$ peut être utilisé pour rendre compte de l'intensité de la réaction, c'est-à-dire de l'importance de la formation des liaisons intergranulaires.

Une méthode simple (Fig. 13) a été mise au point pour permettre la mesure du volume de dioxyde de carbone dégagé, à partir du début de l'opération d'injection.

L'eau distillée a été utilisée pour la saturation des échantillons ainsi que pour la récupération du $\mathrm{CO}_{2}$, ceci pour empêcher toute dissolution partielle dans une eau qui contiendrait des carbonates. Le dioxyde de carbone est recueilli dans une éprouvette graduée.

Pour tous les échantillons testés la réaction se poursuit au-delà d'une heure. Nous avons cependant interrompu la mesure du volume de $\mathrm{CO}_{2}$ quelques minutes avant le début du stockage de l'échantillon dans l'eau qui est programmé une heure après la fin de l'injection.

Les échantillons testés, humides au moment de leur injection, sont ceux utilisés dans l'étude de l'influence de la granulométrie. La figure 14 montre les courbes de dégagement de $\mathrm{CO}_{2}$ en fonction du temps.

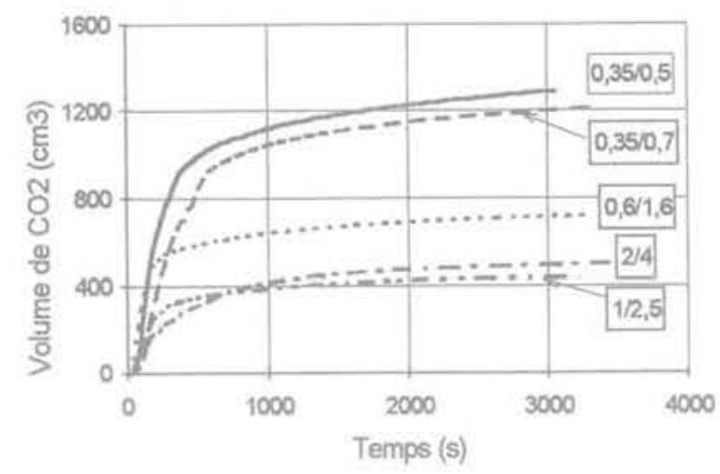

FG. 14 Dégagement de $\mathrm{CO}_{3}$ au cours de la réaction chimique. Echantillons humides de carbonates naturels de différentes granulométries.

Release of $\mathrm{CO}$, during the chemical process. Specimens of wet natural carbonates of different grain size distributions.

Le volume dégagé est plus important pour les sables fins. Ces derniers offrent au produit une plus grande surface de contact que les sables grossiers. La réaction chimique est plus importante et par conséquent, le nombre de liaisons intergranulaires créées est. plus élevé.

Sur la figure 15 , les valeurs du volume de $\mathrm{CO}_{2}$ dégagé à 5 et à 50 minutes du début de la réaction chimique sont regroupées avec les résultats des essais de compression simple, en fonction du diamètre moyen des grains.

Il apparaît sur la figure 15 que les deux paramètres, résistance à la compression simple à 48 heures et volume de $\mathrm{CO}_{2}$ dégagé pendant la réaction chimique, présentent des évolutions similaires.

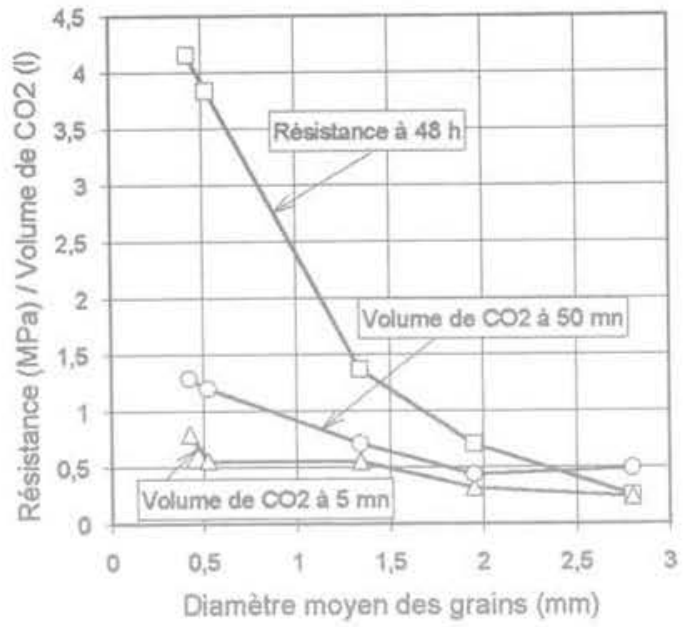

FG, 15 Résistance à la compression simple et volume de $\mathrm{CO}_{4}$ dégagé pendant la réaction chimique, pour des échantillons de carbonates naturels de différentes granulométries.

Unconfined compressive strength and released volume of $\mathrm{CO}$, during the chemical process of natural carbonate specimens of different grain size distributions.

Ces deux paramètres augmentent avec la finesse des crains et donc avec la surface spécifique des matériaux injectés qu'un calcul rapide a permis d'évaluer. Les valeurs ainsi obtenues sont reportées avec les valeurs du volume de $\mathrm{CO}_{2}$ dégagé dans le tableau VI.

\begin{tabular}{|c|c|c|c|c|c|}
\hline & \multicolumn{5}{|c|}{ Granulométrie ( $\mathrm{mm}$ ) } \\
\hline & $0,35 / 0,5$ & $0,35 / 0,7$ & $0,6 / 1,6$ & $1 / 2,5$ & $2 / 4$ \\
\hline$s\left(m^{2} / \mathrm{kg}\right)$ & 5,49 & 4,65 & 2,39 & 1,45 & 0,81 \\
\hline$v\left(\mathrm{~cm}^{3}\right)$ & 1300 & 1200 & 700 & 425 & 500 \\
\hline
\end{tabular}

$\mathrm{s}$ : surface spécifique, $\mathrm{v}$ : volume de $\mathrm{CO}_{2}$ -

\section{6}

\section{Résistance en fonction de la vitesse de déformation}

Afin de déterminer l'influence de la vitesse de déformation, une série d'essais de compression simple et de fluage a été réalisée. Les échantillons, préparés à partir du sable de Manche de granulométrie 0,315/0,63 mm, ont été injectés à l'êtat humide (degré de saturation de l'ordre de $78,5 \%$ ).

La teneur en extrait sec du produit ètait de $20 \%$. Le tableau VII précise les conditions de réalisation de ces essais. La figure 16 regroupe les courbes contraintedéformation obtenues au cours de l'essai de compression simple. La résistance diminue pour des vitesses de déformation plus faibles. L'écart de résistance observé est de l'ordre de 12 à $15 \%$ lorsque le rapport des vitesses est voisin de 10. Les courbes montrent que la variation du module de résistance est peu importante. 


\begin{tabular}{|c|c|c|c|c|c|c|c|c|c|}
\hline Numéro de l'essai & 26 & 27 & 28 & 29 & 30 & 31 & 32 & 33 & 34 \\
\hline Type d'essai & \multicolumn{5}{|c|}{ Compression simple } & \multicolumn{4}{|c|}{ Fluage } \\
\hline $\begin{array}{l}\text { Poids volumique sec moyen } \\
\text { avant injection }\left(\mathrm{kN} / \mathrm{m}^{3}\right)\end{array}$ & 13,4 & 13,4 & 13,4 & 13,4 & 13,4 & 13,4 & 13,4 & 13,4 & 13,4 \\
\hline Granulométrie (mm) & \multicolumn{9}{|c|}{$0,315 / 0,63$} \\
\hline Indice des vides e & 1,02 & 1,02 & 1,02 & 1,02 & 1,02 & 1,02 & 1,02 & 1,02 & 1,02 \\
\hline Degré de saturation (\%) & \multicolumn{9}{|c|}{$78,5 \pm 0,5$} \\
\hline Extrait sec du produit ES (\%) & \multicolumn{9}{|c|}{20} \\
\hline Pression d'injection (kPa) & 210 & 230 & 230 & 220 & 230 & 230 & 230 & 230 & 230 \\
\hline Volume injecté $\left(\mathrm{cm}^{3}\right)$ & \multicolumn{9}{|c|}{$36 \pm 2$} \\
\hline $\begin{array}{l}\text { Temps de maintien } \\
\text { dans le moule (mn) }\end{array}$ & \multicolumn{9}{|c|}{60} \\
\hline $\begin{array}{l}\text { Durée de stockage } \\
\text { avant essai (heures) }\end{array}$ & \multicolumn{9}{|c|}{48} \\
\hline Température de stockage $\left({ }^{\circ} \mathrm{C}\right)$ & \multicolumn{9}{|c|}{23,5} \\
\hline Charge de fluage (MPa) & \multicolumn{5}{|c|}{1} & 0,4 & 0,8 & 1,2 & 1,6 \\
\hline Vitesse de déformation ( $\mathrm{mm} / \mathrm{mn}$ ) & 0,76 & 0,1 & 0,076 & 0,045 & 0,012 & 0 & 0 & 0 & 0 \\
\hline $\begin{array}{l}\text { Résistance à la compression } \\
\text { simple (MPa) }\end{array}$ & 2,18 & 1,98 & 1,94 & 1,75 & 1.70 & \multicolumn{4}{|c|}{1} \\
\hline Déformation à la rupture (\%) & 1,45 & 1,30 & 1,30 & 1,25 & 1,25 & \multicolumn{4}{|c|}{1} \\
\hline
\end{tabular}

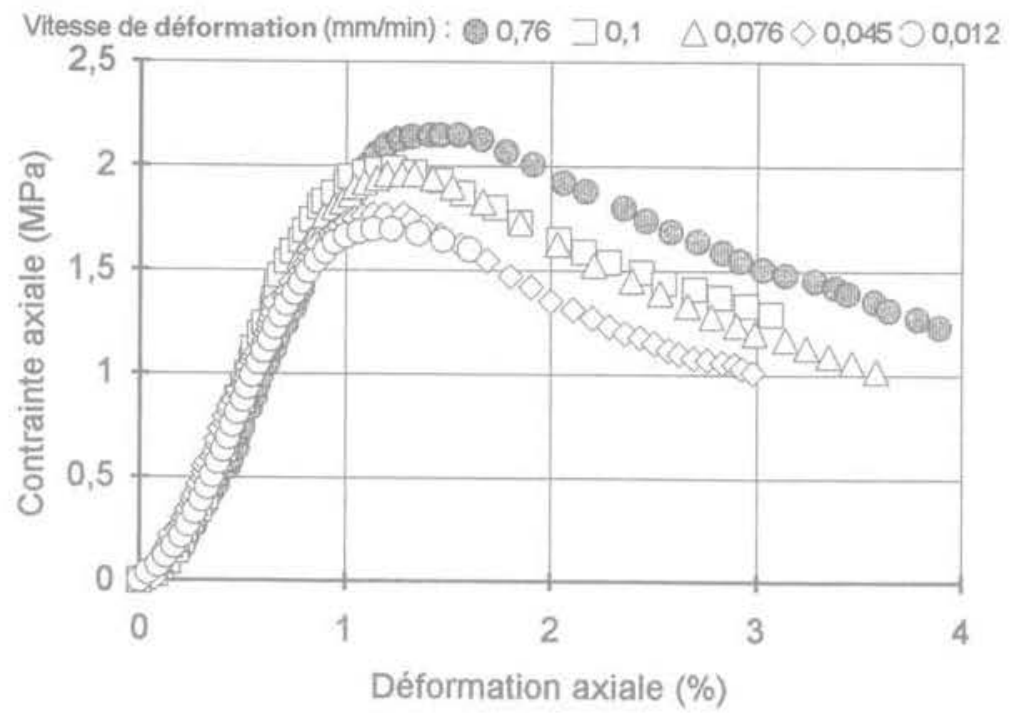

FG. 16 Courbes contrainte-déformation en fonction de la vitesse de déformation. Stress-strain curves versus the strain rate.

La résistance à la compression simple diminuant avec la vitesse de déformation, il devient important de savoir s'il y a une limite de réduction de cette résistance ou si celle-ci continue à diminuer indéfiniment avec le temps. Des éléments de réponse à cette question peuvent être obtenus en combinant les résultats d'essais de fluage avec ceux des essais de compression simple. Les résultats de quatre essais de fluage réalisés sur des éprouvettes similaires à celles des essais de compression simple (tableau VII) sont montrés sur la figure 17. Pour toutes les éprouvettes, il est observé un fluage avec le temps, la déformation de fluage dépendant de la charge appliquée.

Exprimé en pourcentage, le rapport de cette charge à la charge de rupture obtenue à l'essai de compression simple à la vitesse de $0,1 \mathrm{~mm} / \mathrm{min}(0,0011 \% / \mathrm{min})$ 


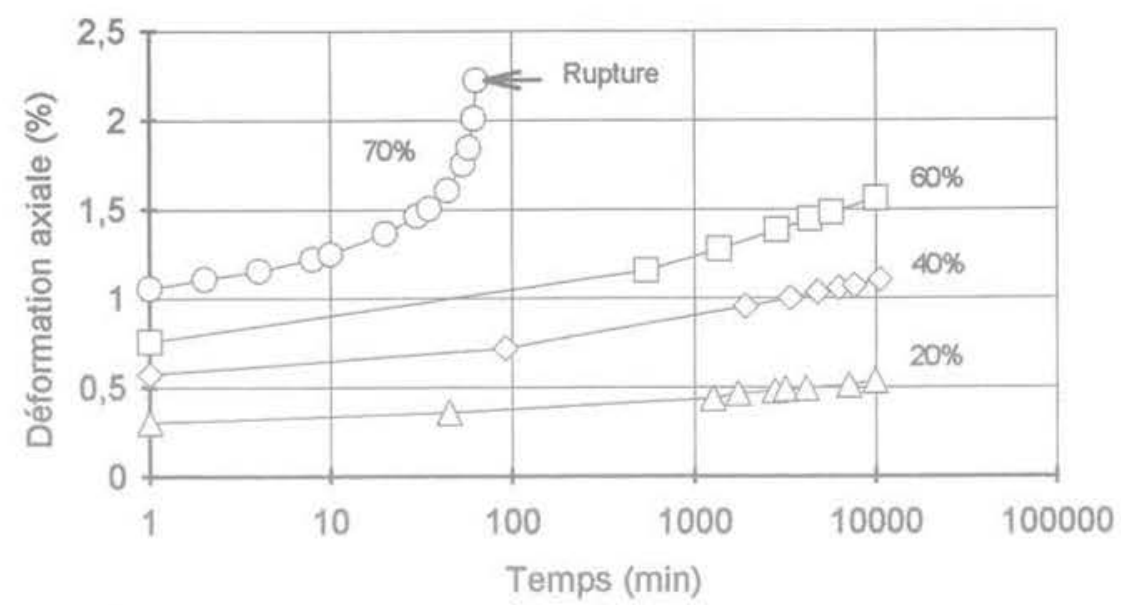

FG: i] Courbes de fluage.

Creep curves.

est indiqué sur la figure. La courbe d'un cinquième essai de fluage réalisé avec une charge de $80 \%$ $(1,6 \mathrm{MPa})$ n'a pu être représentée, la rupture ayant été quasi immédiate. Les résultats de ces essais de fluage montrent que les éprouvettes soumises à un niveau de chargement supérieur ou égal à $70 \%$ se déforment jusqu'à la rupture, celles soumises à un niveau de chargement inférieur à $70 \%$ subissent un fluage mais n'atteignent pas la rupture, tout au moins dans la durée de l'essai (7 jours).

\subsection{7.}

Résistance en fonction de la forme des particules des matériaux carbonatés

La résistance obtenue en compression simple diffère selon la forme des particules des matériaux injectés. Pour le montrer, des essais ont été réalisés avec le sable de Manche (notation SM) et avec les carbonates naturels (notation $\mathrm{CN}$ ) obtenus par concassage d'une roche carbonatée pure.

Pour ces essais, les matériaux considérés possèdent la même granulométrie : $0,35 / 0,7$ et $0,7 / 1,6 \mathrm{~mm}$ pour chaque matériau.

Les particules du sable de Manche, débris de squelettes carbonatés et de coquilles, sont souvent plates et allongées, celles obtenues par concassage de roches sont également anguleuses mais beaucoup plus ramassées.

La figure 18 montre les courbes « contrainte-déformation » obtenues en compression simple pour ces deux types de matériau. Les échantillons sont injectés à l'état sec avec un produit de $15 \%$ d'extrait sec. Les tendances qui apparaissent sur cette figure concernent la résistance maximale et la résistance résiduelle de ces échantillons. La résistance obtenue pour les carbonates naturels est plus grande et la rupture est de type fragile.

A l'inverse, dans le cas du sable de Manche, avec un sommet nettement plus arrondi des courbes

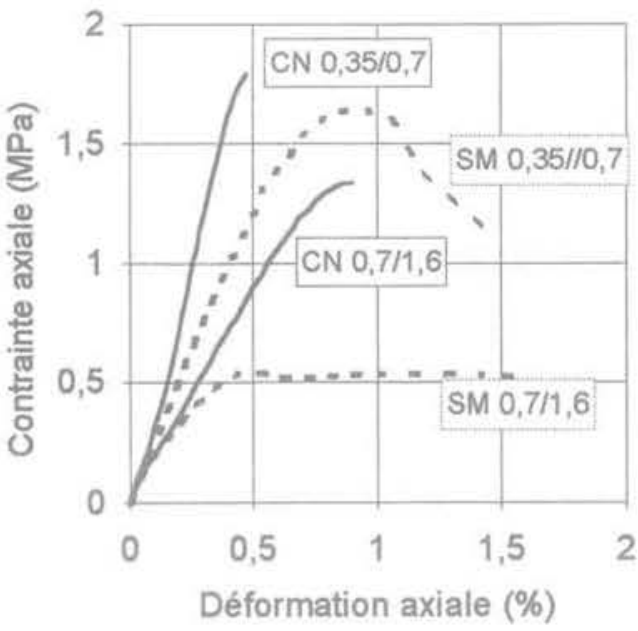

FG, 18 Courbes contrainte-déformation en fonction de la forme des particules. Effect of particles shape on stress-strain curves.

contrainte-déformation, surtout pour une granulométrie plus grossière, la rupture est de type plastique,

La teneur en carbonates de ces deux matériaux est légèrement différente (90\% pour le sable de Manche et près de $100 \%$ pour les carbonates naturels), mais cette différence ne peut pas expliquer à elle seule cet écart entre les résistances maximales. Ce résultat serait plutôt lié au nombre de contacts intergranulaires au sein de l'échantillon qui doit être plus important dans le cas des carbonates naturels où les particules sont plus ramassées. Ceci explique également la plus grande rigidité de ces mêmes matériaux, constatée d'après la pente des courbes obtenues. Quant à la résistance résiduelle, elle peut résulter de la différence de rugosité au niveau de la surface de rupture qui doit être plus importante dans le cas du sable de Manche dont les particules sont plus allongées. 


\section{Conclusions}

Les principales conclusions concernant cette étude de caractérisation des matériaux injectés sont les suivantes:

1. L'amélioration des caractéristiques mécaniques des sols étudiés (sables carbonatés), due à l'injection du produit polymère, est dans tous les cas étudiés très significative. Les valeurs de la résistance à la compression simple d'éprouvettes de sable injecté, fonction de nombreux paramètres, sont de l'ordre de $0,5 \mathrm{MPa}$ quelques dizaines de minutes après l'ínjection, à environ $4 \mathrm{MPa}$ après une conservation de 24 heures à 3 mois dans l'eau à $20^{\circ} \mathrm{C}$ (des essais réalisés à 16 mois de stockage ont donné des valeurs légèrement supérieures). Nous avons également noté la rapidité du développement de cette résistance.

2. Les échantillons initialement denses sont plus résistants et plus rigides que les échantillons injectés à l'état lâche.
3. Une injection réalisée sous faible pression permet un enrobage correct des grains. Il en résulte un enchevêtrement plus dense des macromolécules au sein de l'échantillon et donc une plus grande résistance.

4. Les plus grandes résistances sont obtenues pour des granulométries fines. Lorsque la dimension des grains diminue, la surface spécifique et le nombre de contacts intergranulaires augmentent, permettant à la réaction chimique de créer un plus grand nombre de liaisons intergranulaires et conduisent à une meilleure résistance:

5. Le développement des liaisons intergranulaires et donc de la résistance peuvent être favorisés ou non suivant la forme des particules. Ce développement est plus important dans le cas de particules "plus rondes »). De la forme des particules dépend également la rugosité de la surface de rupture des échantillons. Dans le cas des particules allongées (cas du sable de Manche), la rugosité et par conséquent la résistance résiduelle sont plus élevées.

6. La résistance à la compression simple croît avec la vitesse de déformation choisie. L'accroissement est de l'ordre de $15 \%$ lorsque la vitesse est décuplée.

\section{Bibliographie}

[1] Levacher D., Bennabi A. - Injection des pieux battus dans les sables carbonatés, Annales de l'TTBTP, n 489, 1990, pp. 5872.

[2] Bennabi A. - Application d'un produit polymère au traitement des sols et à l'injection des pieux battus dans les sables, Thèse de doctorat Ecole Centrale de Nantes, Université de Nantes, 1993, $217 \mathrm{p}$.
[3] Groupement Technique d'Ingénierie, 79, RN 10, 78310 Coignières.

[4] OMYA SA, 35, quai André-Citroën. 75725 Paris, 